\title{
Aplicación del software Microsoft Power BI como un sistema de inteligencia artificial \& machine learning en la toma de decisiones y herramienta de tabulación de datos aplicado a la Facultad de CIYA de la
} Universidad Técnica de Cotopaxi en el periodo 2015 2019

(c) (i) () ()

Application of Microsoft Power BI software as an artificial intelligence \& machine learning system in decision making and data tabulation tool applied to the CIYA Faculty of the Technical University of Cotopaxi in the period 2015 - 2019

Víctor Hugo Medina Matute. ${ }^{1}$, Lando Stephen Ocaña Pañora. ${ }^{2}$ Mario Agustín Banda Casa. ${ }^{3} \&$ Mirella Nataly Arias Guadalupe. ${ }^{4}$

Abstract.

DOI: https://doi.org/10.33262/concienciadigital.v4i3.1.1834

Introduction. The data generated by the Technical University of Cotopaxi in the different academic and administrative areas have several cases that lead to establish the situation

\footnotetext{
${ }^{1}$ Universidad Técnica de Cotopaxi, Latacunga, Ecuador, victormedina@utc.edu.ec, https://orcid.org/00000002-6149-453X

2 Universidad de las Fuerzas Armadas "ESPE”, Latacunga, Ecuador, 1socana@espe.edu.ec, https://orcid.org/0000-0002-4748-5282

${ }^{3}$ Universidad Técnica de Cotopaxi, Latacunga, Ecuador, mario.banda@utc.edu.ec, https://orcid.org/00000003-3950-1286

${ }^{4}$ Investigador Independiente, Riobamba, Ecuador, mirella.arias1994@ hotmail.com, https://orcid.org/00000001-7643-2633
} 
of the students' qualifications. Target. The implementation of data mining is suggested to obtain an analysis of student performance applied in engineering careers at the Faculty of Engineering and Applied Sciences, FCIYA that allows efficient information management for decision-making by authorities. Methodology. An intelligence method is established for the management, processing and analysis of the data that will help to generate reports of qualifications of the students of the faculty with a high level of veracity and timely to support in the making of managerial decisions. The tool chosen to use is Microsoft Power BI, considered one of the most successful, it will evaluate the averages of the academic cycles from September 2015 to February 2019 to verify the career with the greatest learning deficit. Results. After the analysis, it was possible to determine that the Electromechanical Engineering career presents the greatest learning deficit, obtaining low averages in all the comparison criteria used in the software for the evaluation of said item compared to the other careers of the FCIYA.

Keywords: Artificial Intelligence, Microsoft Power BI, data mining, academic deficit, decision tool.

\section{Resumen.}

Introducción. Los datos que genera la Universidad Técnica de Cotopaxi en las diferentes áreas académicas y administrativas tienen varios casos que conllevan a establecerla situación de las calificaciones de los estudiantes. Objetivo. Se sugiere la implementación de una minería de datos para obtener un análisis del desempeño estudiantil aplicado en carreras de ingeniería en la Facultada de Ciencias de la Ingeniería y Aplicadas, FCIYA que permita un manejo de la información eficiente para la toma de decisiones de las autoridades. Metodología. Se establece un método de inteligencia para el manejo, procesamiento y análisis de los datos que sirvan de ayuda a la generación de informes de calificaciones de los estudiantes de la facultad con un alto nivel de veracidad y oportuna para el apoyo en la toma de decisiones gerenciales. La herramienta escogida para emplear es Microsoft Power BI, considerada como una de las más exitosas, evaluará los promedios de los ciclos académicos de septiembre 2015 a febrero de 2019 para verificar la carrera con mayor déficit de aprendizaje. Resultados. Posterior al análisis se logró determinar que la carrera de Ingeniería en Electromecánica presenta el mayor déficit de aprendizaje, obteniendo promedios bajos en todos los criterios de comparación empleados en el software para la evaluación de dicho rubro comparado con las demás carreras de la FCIYA.

Palabras claves: Inteligencia Artificial, Microsoft Power BI, minería de datos, déficit académico, herramienta de decisión.

\section{Introducción.}

La facultad de CIYA de la Universidad Técnica de Cotopaxi atraviesa un problema en el análisis de datos enfocados en específico a las materias de las carreras técnicas. No se 
aplican métodos, estrategias o técnicas para el análisis de datos, existe una falta de capacitación en la forma de impartir sus clases, esto tiene repercusión directa en el aprendizaje de los estudiantes y conlleva a un bajo desempeño notorio.

En este estudio se toma en cuenta esta problemática como propuesta directa a investigar e intervenir de la siguiente manera:

La problemática tiende a su aumento cuando se analiza las irregularidades en el sistema educativo, las cuales pueden aumentar drásticamente la dificultad, se ven reflejadas en el interés del estudiante como también en relación directa con las cualidades y nivel del docente.

La Facultad de Ciencias de la Ingeniería y Aplicadas (FCIYA) de la Universidad Técnica de Cotopaxi día a día genera una gran cantidad de datos de los diferentes campos académicos y administrativos los cuales no cuentan con una técnica o sistema de manejo de información que podrían ser usados para la toma de decisiones.

El problema surge en el cómo beneficiar a la Universidad Técnica de Cotopaxi mediante el uso de alguna herramienta de decisión que pueda entregar resultados para una toma de decisiones haciendo uso de los datos almacenados.

Como objetivo principal de la investigación se tiene el determinar un método con inteligencia en toma de decisiones con base a la herramienta Power BI, enfocado al manejo, procesamiento y análisis de datos de rendimiento académico de la Universidad Técnica de Cotopaxi en la Facultad de Ciencias de la Ingeniería y Aplicadas.

Mediante el empleo de Microsoft Power BI se podrá evaluar los promedios registrados en los ciclos académicos de rango septiembre 2015 a febrero 2019, con el fin de corroborar cual carrera tiene un mayor déficit de aprendizaje.

\section{Estado del arte.}

Como estado del arte de esta investigación se presentan algunos trabajos relacionados con el tema propuesto, especialmente en lo que respecta a la Inteligencia de Negocios, Big Data y toma de decisiones.

Estando en plena era de la información, existe la necesidad que las compañías evalúen muy bien sus necesidades con el fin de poder contemplar la adopción de algún sistema de información que la consolide y le brinde la estabilidad que tanto ambicionan. (Fabara \& Zapata, 2015)

La Implementación de una Solución de Inteligencia de Negocios para la Gestión del Talento Humano en la Universidad Técnica del Norte, permite a los gerentes de recursos humano encontrar relaciones entre las personas y los resultados de la organización con mayor eficiencia. Este trabajo requiere el estudio de doce indicadores de talento humano, de los datos registrados en el Sistema Informático Integrado Universitario SIIU, y de las 
herramientas: Oracle Database, Oracle Business Intelligence, Tableau, Oracle Analytics. Es fundamental aprovechar la gran cantidad de información registrada en el SIIU para tener un panorama global del talento humano.

Estos procesos pueden ser apoyados usando la Inteligencia de Negocios que ofrece la posibilidad de medir negocios, adquirir información que antes no estaba disponible, descubrir patrones de comportamiento, simular y optimizar las actividades de los empleados para el futuro. Además, el tener datos adecuadamente integrados en un almacén de datos permite la implementación de las funciones más importantes del BI. La entrega de valor a los usuarios empresariales debe ser el objetivo principal y no negociable de un sistema de Inteligencia de Negocios (Muryjas \& Waver, 2014).

Las soluciones de BI ayudan a las empresas a ser más eficientes, encontrando áreas puntuales que permitan identificar nuevas oportunidades de negocio. Muchas compañías pierden mucho tiempo buscando información de varias fuentes de datos departamentales, sin embargo, con BI, toda la información está centralizada, siendo accesible visualmente desde un tablero o un informe, lo que permite ahorrar gran cantidad de tiempo (Kriz, 2015. p.45).

Por otra parte, en una encuesta sobre el estado de la analítica de negocios realizada por Bloomberg Businessweek, se obtuvo como resultado que el 97 por ciento de las empresas con ingresos superiores a los 100 millones de dólares utilizaron algún tipo de analítica comercial. Un informe de McKinsey Global Institute predijo que para el 2018, Estados Unidos enfrentará una falta de 140,000 a 190,000 personas con habilidades analíticas, así como un déficit de 1.5 millones de administradores informáticos con el conocimiento para analizar grandes cantidades de datos (Chen, Chiang, \& Storey, 2012).

Analizar herramientas para aplicar técnicas de data analytics y Big data sobre las redes sociales. Innovar en el dictado de materias de años avanzados de la carrera acorde a los nuevos paradigmas educativos y formas de comunicarse de la sociedad. (Molinari, 2016)

Con base en el estudio comparativo generado en la presente investigación se determinó que SPSS de IBM fundamenta y colabora en todas las etapas de la propuesta metodológica planteada generando así un análisis predictivo y confiable, además de generar soluciones inmediatas e interactivas de su rendimiento actual con la capacidad de predecir resultados futuros y actuar proactivamente para el cumplimiento de los mismos. (Espino, 2013)

Como objetivo de este trabajo se pretende la construcción de un marco comparativo que permita confrontar los distintos modelos, y evaluar la adecuación de los mismos en escenarios donde el proyecto de minería de datos tiene por objetivo colaborar en la solución de un problema organizacional. (Trujillo \& Rivas, 2005) 


\section{Fuentes de datos}

Según Hassanifar (2015), las fuentes de datos pueden ser bases de datos relacionales o cualquier otra estructura de datos que soporte la línea de aplicaciones de negocio. Estar alojadas en muchas plataformas diferentes y contener información estructurada, como tablas u hojas de cálculo, o información no estructurada como archivos de texto claro o imágenes y otra información multimedia.

\section{Power BI}

La herramienta Power BI, es una herramienta en la nube. "Es un conjunto de varias herramientas de análisis empresarial, que pone el conocimiento al alcance de toda la organización. Permite el enlace de cientos orígenes de datos diferentes, permite una preparación simple de todos los datos. Brinda una fácil presentación a todo nivel organización de todos los datos e información valiosa para el direccionamiento y control." (Microsoft, 2020).

Entre las principales funciones que tiene este software se pueden mencionar las siguientes:

- Hojas de cálculo en Excel, servicios en la nueve, datos de transmisión y bases de datos locales.

- Manejo de datos en tiempo real.

- Capacidad de respuesta oportuna y eficaz.

- Creación de paneles, informes y conjuntos de datos.

- Permite una misma visión para todos los participantes en cada uno de los procesos de la organización.

- Puede llegar a miles de usuarios con una información actualizada y confiable.

Power BI, es la nueva herramienta de Business Inteligence (BI) que incorpora la suite de productividad, Office 365; por lo que, esta nueva herramienta permite analizar e interactuar con una cantidad masiva de datos dentro de Excel y recabando información a través de lenguaje natural y peticiones del tipo pregunta-respuesta. (Softeng, 2015)

La aplicación Microcrosoft Power es una plataforma de Inteligencia de Negocios (Business Intelligence) orientada a la solución y centrada en procesos que es capaz de ejecutar las reglas de negocio necesarias, expresadas en forma de procesos, actividades, además es capaz de presentar y entregar la información adecuada en el momento adecuado, es una solución de análisis empresarial que permite visualizar los datos y compartir información con toda la organización, o insertarla en su aplicación o sitio web. Conectarse a cientos de orígenes de datos y dar vida a los datos con los paneles e informes dinámicos. (Pérez \& Merino, 2012) 


\section{Características de Microsfot Power BI}

Es característico, debido a que esta herramienta, permite la conexión a cientos de orígenes de datos, con la preparación de datos simplificada, generación de análisis ad hoc; además, esta herramienta de Business Inteligencie permite dar vida a los datos con los paneles e informes dinámicos.

\section{Ventajas de Microsoft Power BI}

El resumen de las ventajas que presenta esta herramienta se resume en la siguiente tabla a continuación.

\section{Tabla 1}

Ventajas del uso de Microsoft Power BI

\begin{tabular}{|c|c|}
\hline \multicolumn{2}{|c|}{ VENTAJAS DE POWER BI } \\
\hline Ventaja & Detalle \\
\hline Flexibilidad & $\begin{array}{l}\text { Permite extraer información } \\
\text { importante para una amplia gama de } \\
\text { escenarios. }\end{array}$ \\
\hline $\begin{array}{l}\text { Optimizar, Limpiar, } \\
\text { transformar y combinar }\end{array}$ & $\begin{array}{l}\text { Estos datos de múltiples orígenes, } \\
\text { permiten analizar en profundidad los } \\
\text { datos y encontrar patrones. }\end{array}$ \\
\hline Innovación. & $\begin{array}{l}\text { Podrás crear informes sorprendentes } \\
\text { con visualizaciones de datos } \\
\text { interactivas. }\end{array}$ \\
\hline Personalizar & $\begin{array}{l}\text { Diseñar tu informe mediante las } \\
\text { herramientas de creación de temas, } \\
\text { formato y diseño. }\end{array}$ \\
\hline Multiplataforma & $\begin{array}{l}\text { Crear informes optimizados para } \\
\text { dispositivos móviles. }\end{array}$ \\
\hline
\end{tabular}

Fuente: Elaboración propia.

\section{Minería de Datos}

La minería de datos se refiere a la extracción o "minería" de conocimientos desde grandes cantidades de datos (Ahmed \& Elaraby, 2014). La minería de datos comprende los algoritmos básicos que permiten obtener conocimientos fundamentales y conocimientos de datos masivos. La minería de datos es parte de un proceso de descubrimiento de conocimiento más amplio, que incluye tareas de pre-procesamiento como extracción de datos, limpieza de datos, fusión de datos, reducción de datos y construcción de características, así como pasos de post-procesamiento interpretación de patrones y modelos, generación y confirmación de hipótesis. Este proceso de descubrimiento de conocimiento y minería de datos tiende a ser altamente interactivo, es decir, el intercambio de información persona computador, la participación entre los usuarios y los sistemas informáticos (Zaki \& Meira, 2014). 


\section{Técnicas de Minería de Datos}

Las técnicas de minería de datos se utilizan para manipular grandes cantidades de datos con el fin de descubrir patrones ocultos y relaciones útiles en la toma de decisiones. Existen algunas técnicas de clasificación: agrupamiento, regresión, inteligencia artificial, redes neuronales, reglas de asociación, árboles de decisión, etc.

El datamining es el término utilizado para señalar al conjunto de técnicas y tecnologías que permiten explorar de forma automática grandes bases de datos, el objetivo central es explorar, analizar, y aplicar el conocimiento (Vlcárce, 2004).

Básicamente, el datamining es una minería de datos cuyo propósito es extraer conocimiento útil y entendible a raíz del análisis de varios datos, mediante regla so patrones significativos, que presenten conocimiento implícito (Rodríguez \& Díaz, 2009).

\section{Inteligencia artificial}

La inteligencia artificial (o IA), es una rama de la ciencia que estudia la inteligencia en objetos artificiales y, desde un punto de vista técnico e ingenieril, plantea la creación de elementos que tengan un comportamiento inteligente. En otras palabras, la IA se fundamenta en la construcción de máquinas y sistemas que tengan un comportamiento similar al que llevaría a cabo una persona. (Romero, 2007)

El aprendizaje, la capacidad de adaptación a entornos cambiantes, la creatividad, etc., son facetas que usualmente se relacionan con el comportamiento inteligente. Por otro lado, esta ciencia es multidisciplinaria, en la que intervienen diferentes disciplinas, tales como la Neurociencia, la Psicología, las Tecnologías de la Información, la Ciencia Cognitiva, la Física, las Matemáticas, etc. (Romero, 2007)

\section{Enfoques de la Inteligencia Artificial}

En este apartado se pueden definir dos enfoques principales de la IA: el punto de vista tecnológico y el punto de vista científico.

Por el lado de la tecnología, la rama ingenieril plantea la creación de sistemas informáticos que ejecuten tareas que requieren un grado de inteligencia humana. Se persigue desde este planteamiento la resolución de problemas en concreto, sin ninguna limitación de ninguna índole. (Romero, 2007)

Por otra parte, la rama científica de la IA puede definirse como el estudio del comportamiento inteligente, teniendo como objetivo principal la consecución de una teoría de la inteligencia que permita explicar la conducta que va a producirse en seres de naturaleza inteligente, guiando la creación de máquinas o sistemas que sean capaces de alcanzar dicha inteligencia. (Romero, 2007) 


\section{Redes de neuronas artificiales}

Estas redes son conocidas también como "Sistemas Conexionistas". Se definen como un sistema de procesamiento de información que se basa en el funcionamiento del sistema nervioso biológico, intentando emular el comportamiento del cerebro humano. (Romero, 2007)

Funcionan como un sistema masivamente paralelo que se ha mostrado eficiente para la resolución inteligente de distintos tipos de problemas. Las RNA constan de elementos básicos de proceso, normalmente organizados en capas (basados en las neuronas biológicas) y con gran cantidad de conexiones entre ellos (basadas en las sinapsis de las neuronas). (Romero, 2007)

\section{Metodologia.}

Para alcanzar el objetivo planteado en el párrafo anterior, se ha decidido el empleo de Microsoft Power BI para evaluar los promedios registrados en los ciclos académicos correspondientes a septiembre de 2015 hasta febrero de 2019, esto con el fin de verificar cuál es la carrera que tiene un mayor déficit en el proceso de aprendizaje. Por lo tanto, se establecen los siguientes objetivos específicos: Emplear la investigación bibliográfica para conocer el estado del arte sobre la inteligencia de negocios y toma de decisiones. Además, Aplicar principios de la metodología de Business Intelligence y Big Data a través del uso de la herramienta de Power BI que permita el análisis de los principales indicadores del rendimiento académico de la Facultad de Ciencias de la ingeniería y Aplicadas (FCIYA). Y Determinar las principales dificultades presentes en los estudiantes de las carreras de la Facultad de Ciencias de la ingeniería y Aplicadas de la Universidad Técnica de Cotopaxi que permita gerenciar estrategias de toma decisiones adecuadas orientadas al mejoramiento del rendimiento académico. (Medina, 2020)

Para la consecución de estos objetivos se plantean las siguientes actividades mostradas en la siguiente tabla.

Tabla 2

Tareas a ejecutar para la consecución de los objetivos planteados

\begin{tabular}{lll}
\hline \multicolumn{1}{c}{ Objetivo } & \multicolumn{3}{c}{ Actividad (Tareas) } \\
\hline & $\begin{array}{l}\text { Buscar información en fuentes confiables. } \\
\text { Analizar las temáticas para la } \\
\text { investigación. } \\
\text { 1. Objetivo específico: Emplear la } \\
\text { investigación bibliográfica para conocer el } \\
\text { estado del arte sobre la inteligencia de } \\
\text { negocios y toma de decisiones. }\end{array}$ & $\begin{array}{l}\text { Aplicar el conocimiento obtenido en la } \\
\text { investigación. } \\
\text { Revisión de la literatura con relación a la } \\
\text { herramienta de decisión y su aplicación }\end{array}$ \\
&
\end{tabular}

Fuente: Elaboración propia. 


\section{Tabla 2}

Tareas a ejecutar para la consecución de los objetivos planteados (continuación)

Objetivo Actividad (Tareas)

2. Objetivo específico: Aplicar principios de la metodología de Business Intelligence y Big Data a través del uso de la herramienta de Power BI que permita el análisis de los principales indicadores del rendimiento académico de la Facultad de Ciencias de la ingeniería y Aplicadas (FCIYA).

3. Objetivo específico: Determinar las principales dificultades presentes en los estudiantes de las carreras de la Facultad de Ciencias de la ingeniería y Aplicadas de la Universidad Técnica de Cotopaxi que permita gerenciar estrategias de toma decisiones adecuadas orientadas al mejoramiento del rendimiento académico.
Realizar el instrumento de recolección de datos.

Realizar un pilotaje de la Big Data con la metodología cuantitativa

Buscar los datos relevantes para la investigación sobre la dificultad en el aprendizaje

Fuente: Elaboración propia.

Para la consecución de estas tareas, se plantean las etapas propuestas en la siguiente tabla.

Tabla 3

Etapas del proyecto

\begin{tabular}{|c|c|}
\hline Etapa & Descripción \\
\hline $\begin{array}{l}\text { Etapa } 1 . \text { Revisión } \\
\text { tecnológica }\end{array}$ & $\begin{array}{l}\text { En esta etapa se revisará toda la información } \\
\text { correspondiente al proceso de minería de } \\
\text { datos. } \\
\text { Buscar la herramienta de decisión más idónea } \\
\text { para la investigación. } \\
\text { Establecer si Power BI cumple con los } \\
\text { requerimientos necesarios. }\end{array}$ \\
\hline $\begin{array}{c}\text { Etapa } 2 . \\
\text { Recolección de } \\
\text { información }\end{array}$ & $\begin{array}{l}\text { En esta etapa se ejecutarán actividades de } \\
\text { recolección de datos relevantes para la } \\
\text { investigación por medio de la Dirección de } \\
\text { TICs de la Universidad Técnica de Cotopaxi } \\
\text { Establecer los datos que sean necesarios para } \\
\text { la investigación- }\end{array}$ \\
\hline $\begin{array}{l}\text { Etapa } 3 \text {. Análisis de } \\
\text { resultados por medio } \\
\text { de técnicas de } \\
\text { decisión }\end{array}$ & $\begin{array}{l}\text { En esta etapa se procesarán los datos obtenidos } \\
\text { de la etapa anterior usando la herramienta } \\
\text { POWER BI. } \\
\text { Conocer datos relevantes y de gran } \\
\text { importancia para la investigación. }\end{array}$ \\
\hline
\end{tabular}

Fuente: Elaboración propia. 
Tabla 3

Etapas del proyecto (continuación)

\begin{tabular}{cl}
\hline Etapa & Descripción \\
\hline & $\begin{array}{l}\text { En esta etapa se recopilan y tabulan los } \\
\text { resultados obtenidos de las etapas anteriores y } \\
\text { se los compila en un informe final, }\end{array}$ \\
Etapa 4. Elaboración & $\begin{array}{l}\text { Se indica cuáles han sido los hallazgos } \\
\text { del informe final } \\
\text { principales y de mayor importancia que se } \\
\text { encontraron durante la investigación }\end{array}$ \\
\hline
\end{tabular}

Fuente: Elaboración propia.

En esta investigación por manejar una minería de datos, se convierte en cuantitativa porque además implica el uso de herramientas informáticas, estadísticas, y matemáticas para obtener resultados. En consecuencia, se utilizó el método cuantitativo porque es concluyente en su propósito ya que trata de cuantificar el problema de los estudiantes de ingeniería de la FCIYA de la UTC y entender qué tan generalizado está mediante la búsqueda de resultados proyectables a una población mayor, en vista que cada semestre aumenta el número de inscritos y por ende se incrementa la población estudiantil.

Después de la recopilación de datos, otro paso es el proceso de análisis de datos. El análisis de datos estadísticos requiere herramientas sistemáticas y la realización de procesos. Existen muchas herramientas analíticas tales como pruebas-t de muestras independientes, pruebas-t correlacionadas, determinaciones de desviación, estudio de la varianza y análisis de regresión que pueden usarse para obtener resultados de los datos, actualmente existen herramientas que manejan minería de datos y el big data, a través de la inteligencia artificial o la inteligencia de negocios, que permiten evaluar el comportamiento de la información y establecer la conclusiones para la toma de decisiones.

\section{Material y Métodos}

\section{Método de Validación por Criterios de Expertos}

El método de validación por criterios de expertos es una manera cualitativa que permite comprobar la validez de la metodología de business intelligence propuesta, para la validación se presentará en una reunión de forma oral a un grupo de profesionales con experiencia en los procesos académicos de la FCIYA de la UTC y del funcionamiento del sistema luego se llenará una encuesta con los presentes con las siguientes preguntas:

Tabla 4

Código y preguntas

\begin{tabular}{cl}
\hline Código & \multicolumn{1}{c}{ Pregunta } \\
\hline I1 & $\begin{array}{l}\text { ¿Considera que la metodología de Business Intelligence } \\
\text { es consistente? }\end{array}$ \\
\hline
\end{tabular}

Fuente: Elaboración propia. 


\section{Tabla 4}

Código y preguntas

\begin{tabular}{cl}
\hline Código & \multicolumn{1}{c}{ Pregunta } \\
\hline I2 & $\begin{array}{l}\text { ¿Considera que la implementación del sistema de } \\
\text { Business Intelligence propuesta es pertinente para la } \\
\text { FCIYA de la UTC? }\end{array}$ \\
\hline I3 & $\begin{array}{l}\text { ¿Considera económicamente viable la implementación } \\
\text { del sistema de Business Intelligence en la FCIYA de la }\end{array}$ \\
& UTC? \\
\hline I4 & ¿Recomendaría la metodología de Business Intelligence? \\
\hline I5 & $\begin{array}{l}\text { ¿Considera que el sistema de Business Intelligence } \\
\text { entribuirá de manera positiva en la toma de decisiones }\end{array}$ \\
\hline
\end{tabular}

Fuente: Elaboración propia.

\section{Proceso del análisis de la información}

En primer lugar, se realizará la encuesta a los expertos quienes son el Vicedecano de la facultad y los directores Académicos de las carreras de ingeniería de la FCIYA de la UTC con el fin de conocer de primera mano los problemas que estos presentan con relación al aprendizaje. Posterior a eso, se analizarán los promedios finales comprendidos entre septiembre de 2015 a febrero de 2019 mediante el uso de la herramienta POWER BI de Microsoft. (Medina, 2020)

\section{Resultados y Discusión.}

Promedio final de la promoción de septiembre 2015 a febrero del 2016

\section{Figura 1}

Promedio final de la promoción de septiembre 2015 a febrero 2016

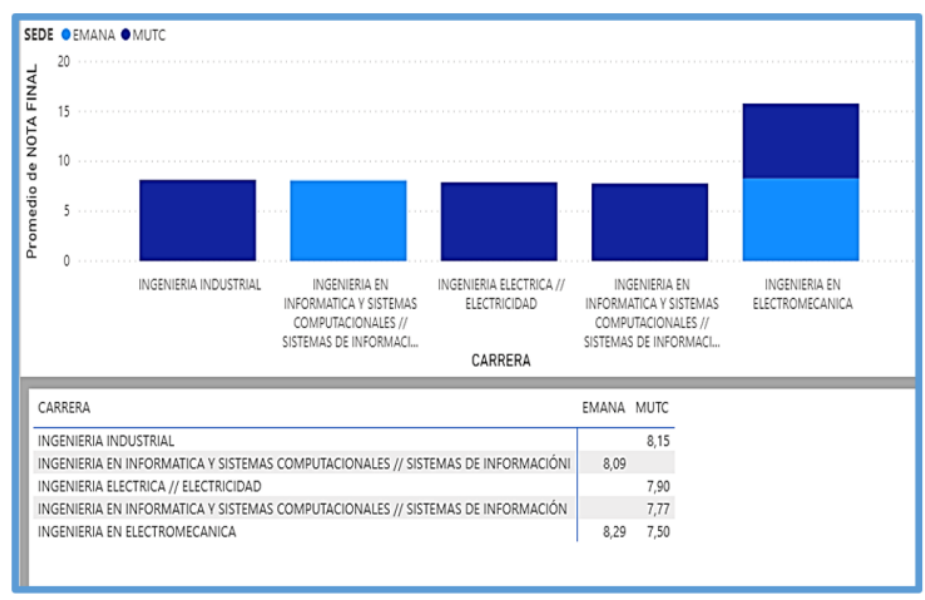

Fuente: Elaboración propia. 


\section{Análisis de resultados}

Como se puede observar en la gráfica, la carrera de Ingeniería en Electromecánica perteneciente a la Matriz de la UTC, presento un promedio general de 7.50, con lo cual se observa un déficit académico con respecto a las otras carreras, pues es la que menor porcentaje alcanzo. (Medina, 2020)

\section{Promedio final de la promoción de abril 2016 a agosto del 2016}

\section{Figura 2}

Promedio final de la promoción abril 2016 a agosto 2016

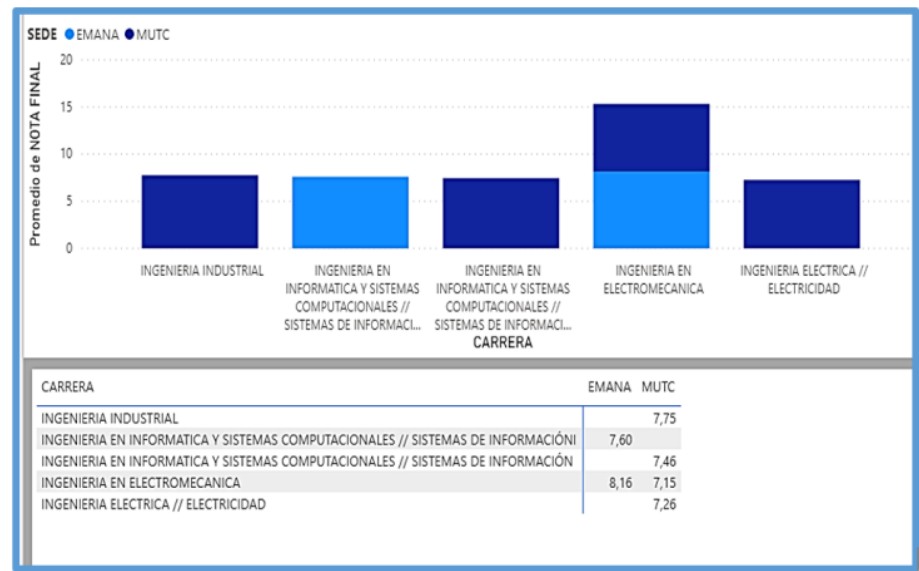

Fuente: Elaboración propia.

\section{Análisis de resultados}

En cuanto a los promedios finales de la promoción abril - agosto 2016, se pudo determinar que, la carrera de Ingeniería en Electromecánica perteneciente a la Matriz de la UTC, obtuvo un promedio general de 7,15 por lo cual, se observa un déficit académico con respecto a las otras carreras. (Medina, 2020)

Promedio final de la promoción de septiembre 2016 a febrero del 2017

\section{Figura 3}

Promedio final de la promoción septiembre 2016 - febrero 2017

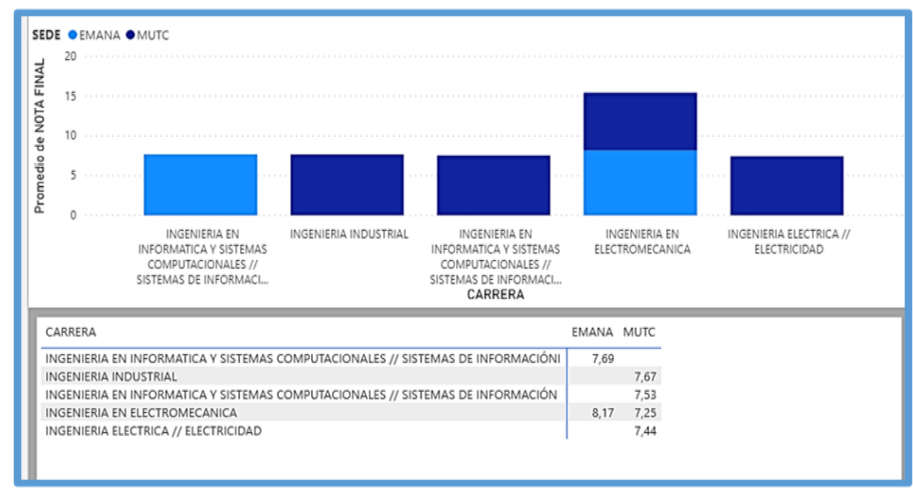

Fuente: Elaboración propia. 


\section{Análisis de resultados}

En lo concerniente a la promoción septiembre 2016 - febrero 2017; se pudo comprobar que la carrera de Ingeniería en Electromecánica perteneciente a la Matriz de la UTC, tiene un promedio general de 7,25 en el cual se observó un déficit académico en relación a otras carreras, puesto que la carrera con mayor puntuación, fue la de Ingeniería en Informática y sistemas computacionales. (Medina, 2020)

\section{Promedio final de la promoción de abril 2017 a agosto del 2017}

\section{Figura 4}

Promedio final de la promoción abril 2017 - agosto 2017

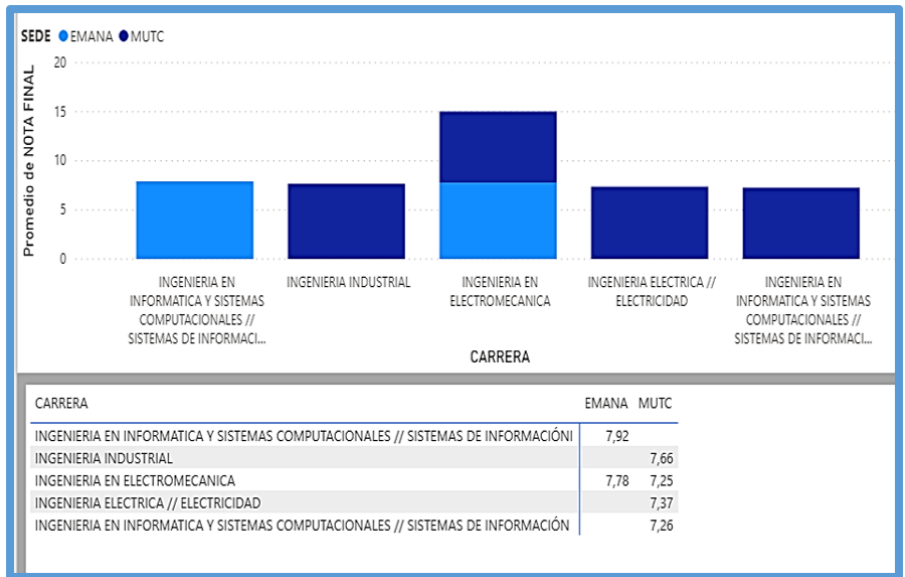

Fuente: Elaboración propia.

\section{Análisis de resultados:}

Por otro lado, la carrera de Ingeniería en Electromecánica, durante el período abril agosto 2017, arrojo un promedio general de 7,25 el cual fue el menor promedio obtenido en la facultad durante ese período; por lo cual presento un déficit académico en respecto con otras carreras. (Medina, 2020) 
Promedio final de la promoción de septiembre 2017 a febrero 2018

\section{Figura 5}

Promedio final de la promoción septiembre 2017 - febrero 2018

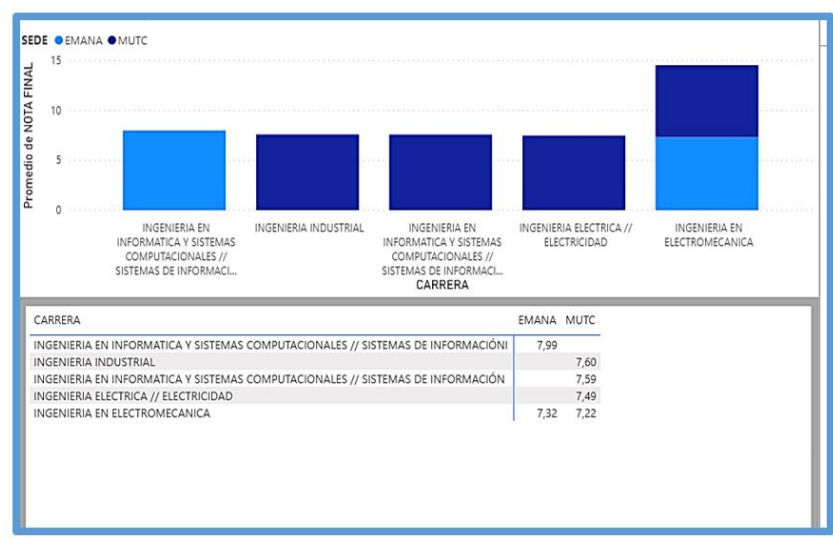

Fuente: Elaboración propia.

\section{Análisis de resultados}

Para la promoción septiembre 2017 - febrero 2018; se pudo corroborar que, la carrera de Ingeniería en Electromecánica perteneciente a la Matriz de la UTC, obtuvo un promedio general de 7,22 el cual determino un déficit académico en respecto con otras carreras. (Medina, 2020)

Promedio final de la promoción de abril 2018 a agosto 2018

\section{Figura 6}

Promedio final de la promoción abril 2018 - agosto 2018

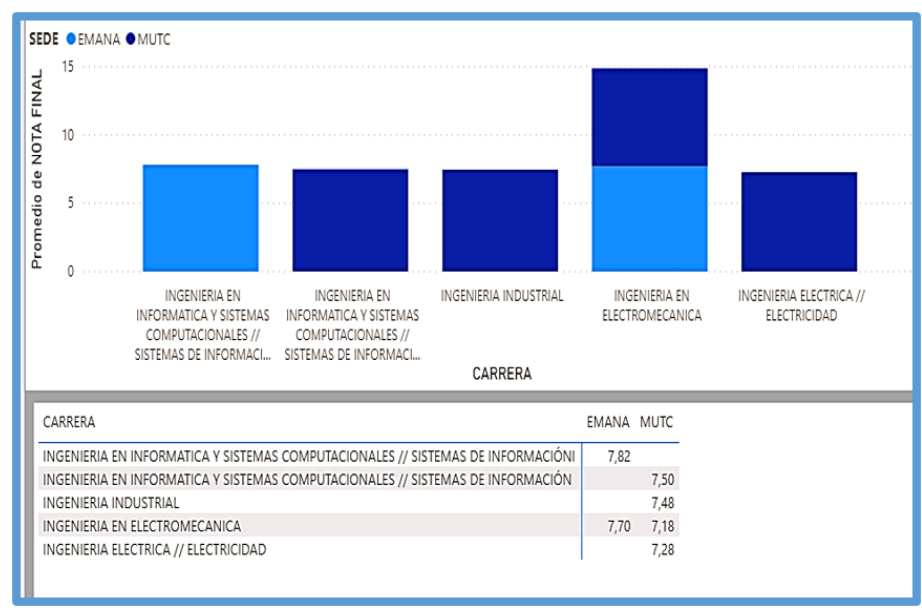

Fuente: Elaboración propia. 


\section{Análisis de resultados}

Durante la promoción de abril-agosto 2018, se pudo comprobar que la carrera de Ingeniería en Electromecánica de la Universidad Técnica de Cotopaxi, tiene un promedio general de 7,18 el cual se observa como un déficit académico, ya que fue el menor promedio con respecto a otras carreras. (Medina, 2020)

\section{Promedio final de la promoción de octubre 2018 a febrero de 2019}

\section{Figura 7}

Promedio final de la promoción octubre 2018 - febrero 2019

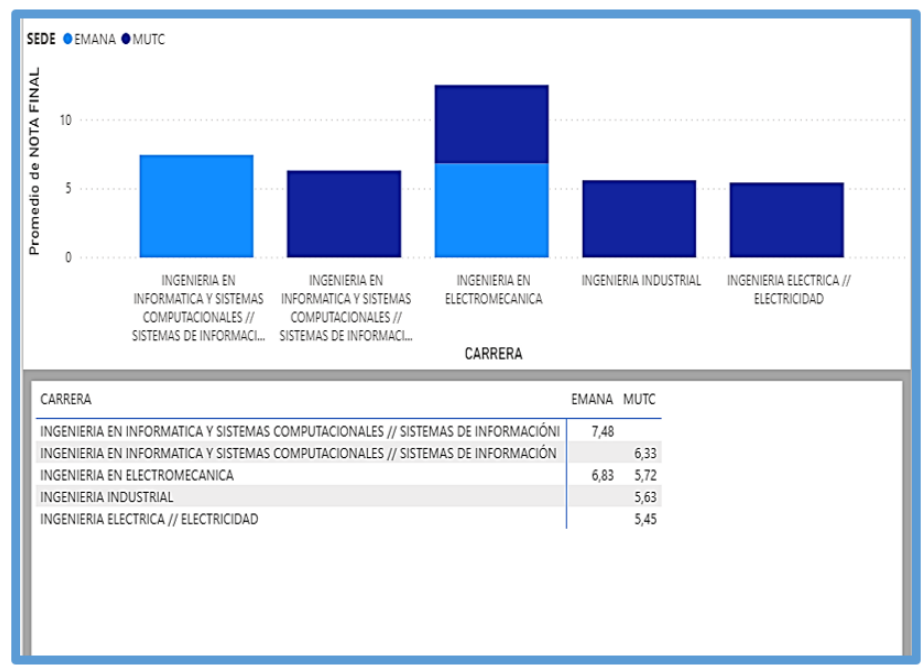

Fuente: Elaboración propia.

\section{Análisis de resultados}

En lo concerniente a la promoción octubre 2018 - febrero 2019; se pudo encontrar que, la carrera de Ingeniería Eléctrica, Electricidad, perteneciente a la matriz de la UTC, obtuvieron un promedio general de 5,45 el cual es inferior a los promedios obtenidos en promociones anteriores, por lo que, se observa como un déficit académico con respecto a otras carreras. (Medina, 2020)

Cuadro comparativo entre carreras del período académico de septiembre 2015 febrero 2019

\section{Tabla 5}

Cuadro comparativo entre carreras del período académico de septiembre 2015 - febrero 2019

\begin{tabular}{|c|c|c|c|c|c|c|c|c|c|c|}
\hline \multicolumn{11}{|c|}{ PERÍODO ACADÉMICO DE SEPTIEMBRE 2015 - FEBRERO 2019} \\
\hline \multirow{4}{*}{ SEDE } & \multirow{4}{*}{ CARRERA } & SEP & $A B R$ & SEP & $A B R$ & SEP & $A B R$ & OCT & \multirow{4}{*}{ PROMEDIO } & \multirow{4}{*}{ Posición } \\
\hline & & 2015 & $2016-$ & 2016 & $2017-$ & 2017 & $2018-$ & 2018- & & \\
\hline & & - FEB & AGOS & - FEB & AGOS & - FEB & AGOS & FEB & & \\
\hline & & 2016 & 2016 & 2017 & 2017 & 2018 & 2018 & 2019 & & \\
\hline EMANA & $\begin{array}{c}\text { INGENIERÍA EN } \\
\text { ELECTROMECÁNICA }\end{array}$ & 8,29 & 8,16 & 8,17 & 7,78 & 7,32 & 7,70 & 6,83 & 7,75 & 2 \\
\hline
\end{tabular}




\section{Tabla 5}

Cuadro comparativo entre carreras del período académico de septiembre

2015 - febrero 2019 (continuación)

\begin{tabular}{|c|c|c|c|c|c|c|c|c|c|c|}
\hline \multicolumn{11}{|c|}{ PERÍODO ACADÉMICO DE SEPTIEMBRE 2015 - FEBRERO 2019} \\
\hline & INGENIERIA EN INFORMATICA Y & & & & & & & & & \\
\hline EMANA & $\begin{array}{l}\text { SISTEMAS COMPUTACIONALES } \\
/ / \text { SISTEMAS DE INFORMACIÓNI }\end{array}$ & 8,9 & 7,60 & 7,69 & 7,92 & 7,99 & 7,82 & 7,48 & 7,91 & 1 \\
\hline MUTC & $\begin{array}{c}\text { INGENIERIA ELECTRICA // } \\
\text { ELECTRICIDAD }\end{array}$ & 7,90 & 7,26 & 7,44 & 7,37 & 7,49 & 7,28 & 5,45 & 7,17 & 5 \\
\hline MUTC & $\begin{array}{c}\text { INGENIERÍA EN } \\
\text { ELECTROMECÁNICA }\end{array}$ & 7,50 & 7,15 & 7,25 & 7,25 & 7,22 & 7,18 & 5,72 & 7,04 & 6 \\
\hline MUTC & $\begin{array}{l}\text { INGENIERIA EN INFORMATICA Y } \\
\text { SISTEMAS COMPUTACIONALES } \\
\text { // SISTEMAS DE INFORMACIÓN }\end{array}$ & 7,77 & 7,46 & 7,53 & 7,26 & 7,59 & 7,50 & 6,33 & 7,35 & 4 \\
\hline MUTC & INGENIERIA INDUSTRIAL & 8,15 & 7,75 & 7,67 & 7,66 & 7,60 & 7,48 & 5,63 & 7,42 & 3 \\
\hline \multicolumn{10}{|c|}{ Promedio Total académico } & 7,44 \\
\hline
\end{tabular}

Fuente: Elaboración propia.

\section{Gráfico comparativo del período académico de septiembre 2015 - febrero 2019}

\section{Figura 8}

Gráfico comparativo del período académico de septiembre 2015 - febrero 2019

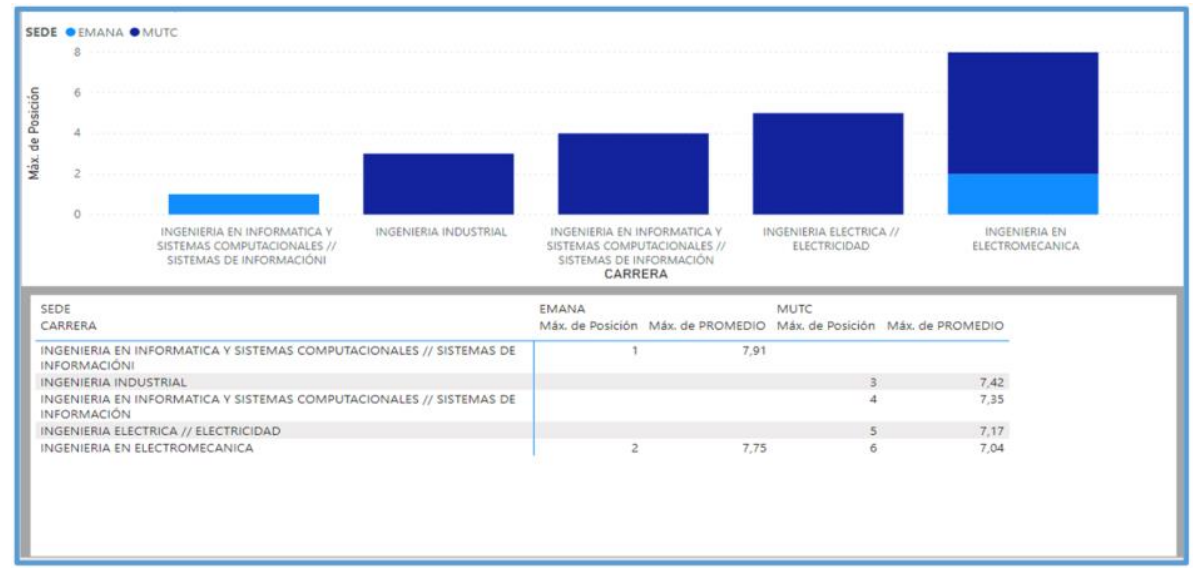

Fuente: Elaboración propia.

\section{Evaluación (Assess)}

Quinta fase, del análisis realizado de la etapa anterior se puede identificar claramente el conocimiento con los resultados obtenidos:

- La carrera de INGENIERÍA EN INFORMÁTICA Y SISTEMAS COMPUTACIONALES // SISTEMAS DE INFORMACIÓN extensión La Mana tiene el mejor promedio académico con un valor de 7,91 desde septiembre 2015 - febrero 2019, logrando una posición de primer lugar.

- La carrera de INGENIERÍA EN ELECTROMECÁNICA extensión La Maná tiene un promedio académico con un valor de 7,75 desde septiembre 2015 - febrero 2019, logrando una posición de segundo lugar. 
- La carrera de INGENIERÍA INDUSTRIAL La Matriz UTC, tiene un promedio académico con un valor de 7,42 desde septiembre 2015 - febrero 2019, logrando una posición de tercer lugar.

- La carrera de INGENIERÍA EN INFORMÁTICA Y SISTEMAS COMPUTACIONALES // SISTEMAS DE INFORMACIÓN La Matriz UTC, tiene un promedio académico con un valor de 7,35 desde septiembre 2015 - febrero 2019, logrando una posición de cuarto lugar.

- La carrera de INGENIERÍA ELÉCTRICA // ELECTRICIDAD La Matriz UTC, tiene un promedio académico con un valor de 7,17 desde septiembre 2015 - febrero 2019, logrando una posición de quinto lugar.

- La carrera de INGENIERÍA EN ELECTROMECÁNICA La Matriz UTC tiene el peor promedio académico con un valor de 7,04 desde septiembre 2015 - febrero 2019, logrando una posición de sexto lugar.

- El promedio general del período académico de septiembre 2015 - febrero 2019 es de 7,44.

- Resultados (Promedio de nota por ciclo de la carrera de INGENIERÍA EN ELECTROMECÁNICA La Matriz UTC período académico de septiembre 2015 - febrero 2019)

- Después del análisis de resultados de Cuadro comparativo del período académico de septiembre 2015 - febrero 2019, se procede analizar el promedio por ciclo de la Carrera de Electromecánica La matriz UTC, el cual tiene un déficit académico.

\section{Conclusiones.}

- Una buena investigación bibliográfica fue de gran ayuda para el desarrollo de esta investigación ya que con una revisión de los antecedentes conceptuales se logró desarrollar el sistema de inteligencia de negocios el cual podría ser usado por la Facultad de Ciencias de la Ingeniería y Aplicadas de la Universidad técnica de Cotopaxi.

- Mediante el análisis realizado en el software Power BI se obtuvo que la carrera de Ingeniería Electromecánica cuenta con un mayor porcentaje de falencia en el proceso de aprendizaje en el periodo académico septiembre 2015 a febrero 2019.

- Mediante la Inteligencia de Negocios o Business Intelligent se puede obtener ventaja para conseguir conocimiento de datos generados en una organización, ayuda de gran manera a generar y fortalecer una toma de decisiones por parte de los directivos.

\section{Recomendaciones.}

- Las nuevas tecnologías son muy importantes para establecer problemas presentes en una organización, cualquier institución debe aplicar una correcta toma de decisiones. 
- Tener en cuenta el uso de una metodología SEMMA ya que es primordial en el desarrollo de Business Intelligent, de no ser así se tornaría una información de libre albedrio y no sería fiable.

- Se debe conocer a fondo el funcionamiento de la herramienta Microsoft Power BI para poder adquirir un manejo eficaz y resultados cercanos a la realidad.

\section{Referencias bibliográficas.}

Chen, H.-c., Chiang, R., \& Storey, V. (2012). Business Intelligence and Analytics: From Big Data to Big Impact. ResearchGate.

Espino, M. (2013). Test de Liderazgo Natural.

Fabara, G., \& Zapata, L. (2015). DISEÑO DE BITS DE INTELIGENCIA PARA EL DESARROLLO Y ESTIMULACIÓN DEL ÁREA DE MATEMÁTICA GENERANDO NUEVOS ESPACIOS EN ELPROCESO ENSEÑANZA APRENDIZAJE EN EL PRIMER AÑO DE EDUCACIÓN BÁSICA DE LA ESCUELA “ISIDRO AYORA” DEL ANTÓN LATACUNGA PROVINCIA DE COTOPAX. 1ATACUNGA, Tungurahua, Ecuador.

Medina, V. (2020). Repositorio Institucional Universidad Técnica de Cotopaxi. Obtenido de http://repositorio.utc.edu.ec/handle/27000/7553

Microsoft. (2020). Microsoft BI. Obtenido de https://powerbi.microsoft.com/eses/features/

Molinari, V. (2016). MEDICIÓN DE INTELIGENCIA. ENTRE DEBATES HISTÓRICOS Y ACTUALES. Anuario de Investigaciones.

Muryjas, P., \& Waver, M. (2014). Business Intelligence as a support in Human Resources strategies realization in contemporary organization. ResearchGate, 65.

Pérez, J., \& Merino, M. (2012). definicion.de. Obtenido de DEFINICIÓN DE LATERALIDAD: https://definicion.de/lateralidad/

Rodríguez, Y., \& Díaz, A. (2009). Herramienta e Minería de Datos. Revista Cubana de Ciencias Informáticas, 73-80.

Romero, J. (2007). INTELIGENCIA ARTIFICIAL Y COMPUTACIÓN AVANZADA. Santiago de Compostela: Fundación Alfredo Brañas.

Softeng. (2015). Softeng. Obtenido de https://www.softeng.es/es-es/blog/power-bi-lanueva-herramienta-de-office-365-para-trabajar-con-datos-masivos.html.

Trujillo, M., \& Rivas, L. (2005). Orígenes, evolución y modelos de inteligencia emocional. Innovar. 
Vlcárce, V. (2004). Data Mining y el descubrimiento del conocimiento. Industrial Data.

Zaki, M., \& Meira, W. (2014). Data Mining and Analysis: Fundamental Concepts and Algorithms. Cambridge University Press.

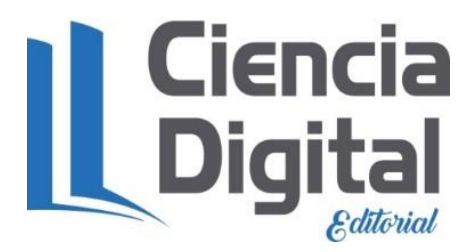




\section{PARA CITAR EL ARTÍCULO INDEXADO.}

Medina Matute, V. H., Ocaña Pañora, L. S., Banda Casa, M. A., \& Arias Guadalupe, M. N. (2021). Aplicación del software Microsoft Power BI como un sistema de inteligencia artificial \& machine learning en la toma de decisiones y herramienta de tabulación de datos aplicado a la Facultad de CIYA de la Universidad Técnica de Cotopaxi en el periodo $2015 \quad$ C ConcienciaDigital, $\quad 4(3.1), \quad 313-332$. https://doi.org/10.33262/concienciadigital.v4i3.1.1834

\section{\Ciencia}

El artículo que se publica es de exclusiva responsabilidad de los autores y no necesariamente reflejan el pensamiento de la Revista Conciencia Digital.

El artículo queda en propiedad de la revista y, por tanto, su publicación parcial y/o total en otro medio tiene que ser autorizado por el director de la Revista Conciencia Digital.

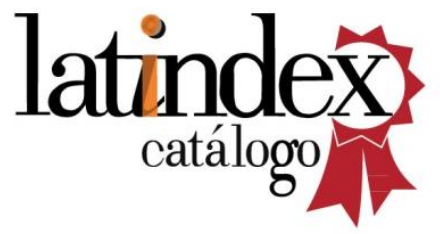

